\title{
Spontaneous perforation of a pyometra presenting as generalized peritonitis
}

\author{
S.W. Hosking \\ Department of Surgery, Royal Postgraduate Medical School, London, W120HS, UK.
}

\begin{abstract}
Summary: Eleven cases of spontaneous perforation of a pyometra have previously been reported. All were associated with, and probably secondary to, cervical occlusion. A further case is described, but differs in that the cervical canal was patent. In the absence of other possible causes of uterine perforation, the aetiology of the perforation in this case remains uncertain.
\end{abstract}

\section{Introduction}

Pyometra is defined as an accumulation of pus in the uterine cavity and has an incidence of approximately $0.5 \%$ of gynaecological inpatients (Henriksen, 1956). Although the aetiology of pyometra is varied, the common pathology to all causes is occlusion of the cervical canal. This may be by benign causes (polyps, atrophic cervicitis) or by malignant causes including both primary tumour and secondary tumours that spread by local invasion (Bostofte \& Legarth, 1981).

Spontaneous perforation of a pyometra and subsequent peritonitis is rare. In 11 cases previously reported, the perforation was always associated with occlusion of the cervical canal and accumulation of pus. This report describes a further case of spontaneous perforation of a pyometra but differs in that the cervical canal was patent. As far as can be ascertained, this has not been previously described.

\section{Case report}

A 77 year old woman was admitted as an emergency, having developed sudden lower abdominal pain $2 \mathrm{~d}$ previously. The pain had become progressively worse, was constant in nature and accompanied by nausea. Prior to this, she had been well apart from a stroke and anteriosclerotic Parkinson's disease. On examination she was pyrexial and had obvious peritonitis. Rectal examination was normal.

At operation, green pus filled the lower abdomen and pelvis. This was from a $3 \mathrm{~cm}$ diameter necrotic area at the uterine fundus which was found to be

Correspondence and present address: S.W. Hosking, F.R.C.S. University Surgical Unit, Royal Hallamshire Hospital, Sheffield S10 2JF, UK.

Accepted: 11 December 1984 perforated and covered by adherent oment $\mathrm{um}$. The uterus was normal in size. At the time, the cause of the perforation was thought to be due to carcinoma or sarcoma of the uterus. There was no evidence of colonic disease. A total hysterectomy and bilateral salpingo-oophorectomy was commenced but before the procedure was completed, the patient suffered a myocardial infarct and died on the operating table. The uterus, ovaries and fallopian tubes were later removed at post-mortem (Figure 1). Bacteriological culture of the pus grew $E$. coli and Bacteroides fragilis. Histological examination of the post mortem specimen showed a small focus of endometrial carcinoma in only one section with no evidence of myometrial invasion. Acute inflammatory changes

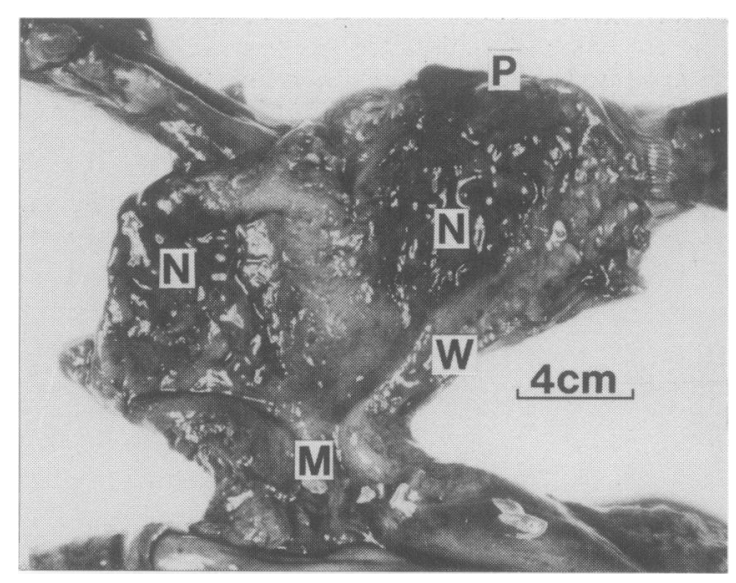

Figure 1 The removed uterus sectioned coronally, showing a necrotic area $(\mathrm{N})$ containing the perforation (P). A marker (M) is in the cervical canal and the uterine wall is labelled $(\mathrm{W})$.

(C) The Fellowship of Postgraduate Medicine, 1985 
were found in the endometrium and myometrium. The cervix was normal.

\section{Discussion}

Uterine pathology leading to peritonitis is uncommon in the elderly patient. Pyometra associated with primary or secondary uterine malignancies is well recognized (Carter et al., 1951), but subsequent spontaneous perforation has previously been described in only 11 patients. Cervical occlusion was present in all cases; in 9 it was secondary to tumour with perforation at a remote site (Bostofte \& Legarth, 1981: Henriksen, 1956), and in two it was due to benign cervical stenosis

\section{References}

BOSTOFTE, E. \& LEGARTH, J. (1981). Spontaneous perforation of pyometra with diffuse peritonitis. Acta Obstetrica et Gynecologica Scandinavica, 60, 511.

CARTER, B., JONES, C.P., ROSS, R.A. \& THOMAS, W.L. (1951). A bacteriological and clinical study of pyometra. American Journal of Obstetrics and Gynecology, 62(4), 793.

CRISP, W.J.C. \& CLAIN, A. (1949). Ruptured pyometra as a
(Loup, 1973; Crisp \& Clain, 1949). In this patient, there was no cervical occlusion and the small focus of uterine adenocarcinoma was thought to be of no significance in the aetiology of the perforation. Other causes of perforation such as recent uterine instrumentation were denied and self instrumentation seemed unlikely. In the absence of cervical occlusion, the aetiology of the uterine perforation described in this patient remains uncertain.

\section{Acknowledgements}

I would like to thank Mr C.B. Wood for allowing me to report this patient who was under his care, and $\mathrm{Mr} \mathrm{K}$. Lim for advice in the preparation of this paper.

surgical emergency. Journal of Obstetrics and Gynecology, 56, 237.

HENRIKSEN, E. (1956). Pyometra associated with malignant lesions of the cervix and uterus. American Journal of Obstetrics and Gynecology, 72, 884.

LOUP, J. (1973). Perforation uterine par pyometrie avec necrose. Journal de Chirurgie (Paris), 105(3), 303. 